\title{
Effects of cross-link breakers, glycation inhibitors and insulin sensitisers on HDL function and the non-enzymatic glycation of apolipoprotein A-I
}

\author{
E. Nobécourt • J. Zeng • M. J. Davies • B. E. Brown • \\ S. Yadav $\cdot$ P. J. Barter $\cdot$ K.-A. Rye
}

Received: 15 August 2007 / Accepted: 22 February 2008 / Published online: 24 April 2008

(C) Springer-Verlag 2008

\begin{abstract}
Aims/hypothesis Hyperglycaemia, a key feature of diabetes, is associated with non-enzymatic glycation of plasma proteins. We have shown previously that the reactive $\alpha$ oxoaldehyde, methylglyoxal, non-enzymatically glycates apolipoprotein (Apo)A-I, the main apolipoprotein of HDL, and prevents it from activating lecithin:cholesterol acyltransferase (LCAT), the enzyme that generates almost all of the cholesteryl esters in plasma. This study investigates whether the glycation inhibitors aminoguanidine and pyridoxamine, the insulin sensitiser metformin and the cross-link breaker alagebrium can inhibit and/or reverse the methylglyoxal-mediated glycation of ApoA-I and whether these changes can preserve or restore the ability of ApoA-I to activate LCAT.

Methods Inhibition of ApoA-I glycation was assessed by incubating aminoguanidine, pyridoxamine, metformin and
\end{abstract}

E. Nobécourt $\cdot$ S. Yadav $\cdot$ P. J. Barter $\cdot$ K.-A. Rye $(\bowtie)$

Lipid Research Group, The Heart Research Institute,

145 Missenden Road, Camperdown,

Sydney, NSW 2050, Australia

e-mail: ryek@hri.org.au

J. Zeng $\cdot$ M. J. Davies · B. E. Brown

Free Radical Group, The Heart Research Institute,

Camperdown,

Sydney, NSW, Australia

M. J. Davies $\cdot$ P. J. Barter $\cdot$ K.-A. Rye

Department of Medicine, University of Sydney,

Sydney, NSW, Australia

K.-A. Rye

Department of Medicine, University of Melbourne,

VIC, Australia alagebrium with mixtures of methylglyoxal and discoidal reconstituted HDL (rHDL) containing phosphatidylcholine and ApoA-I, ([A-I]rHDL). Glycation was assessed as the modification of ApoA-I arginine, lysine and tryptophan residues, and by the extent of ApoA-I cross-linking. The reversal of ApoA-I glycation was investigated by preincubating discoidal (A-I)rHDL with methylglyoxal, then incubating the modified rHDL with aminoguanidine, pyridoxamine or alagebrium.

Results Aminoguanidine, pyridoxamine, metformin and alagebrium all decreased the methylglyoxal-mediated glycation of the ApoA-I in discoidal rHDL and conserved the ability of the particles to act as substrates for LCAT. However, neither aminoguanidine, pyridoxamine nor alagebrium could reverse the glycation of ApoA-I or restore its ability to activate LCAT.

Conclusions/interpretation Glycation inhibitors, insulin sensitisers and cross-link breakers are important for preserving normal HDL function in diabetes.

Keywords Alagebrium · Aminoguanidine - ApoA-I · Apolipoprotein A-I · Glycation · HDL · LCAT · Lecithin: cholesterol acyltransferase $\cdot$ Metformin $\cdot$ Pyridoxamine

$\begin{array}{ll}\begin{array}{l}\text { Abbreviations } \\ \text { (A-I)rHDL }\end{array} & \begin{array}{l}\text { reconstituted HDL containing phosphatidyl- } \\ \text { choline and ApoA-I } \\ \text { apolipoprotein A-I }\end{array} \\ \text { ApoA-I } & \text { Michaelis-Menten constant } \\ K_{\mathrm{m}} & \text { lecithin:cholesterol acyltransferase } \\ \text { LCAT } & \text { 1-palmitoyl-2-oleoyl phosphatidylcholine } \\ \text { POPC } & \text { reconstituted HDL } \\ \text { rHDL } & \text { unesterified cholesterol } \\ \text { UC } & \text { maximum rate } \\ V_{\max } & \end{array}$




\section{Introduction}

Compelling evidence from epidemiological studies indicates that hyperglycaemia is an independent risk factor for cardiovascular disease and that this risk is reduced in patients with diabetes in whom strict glycaemic control is maintained [1]. Results from cross-sectional and prospective studies have indicated that AGE formation is linked to the high prevalence of micro- and macrovascular complications associated with diabetes $[2,3]$.

AGE are generated under conditions of persistent hyperglycaemia when metabolites of reducing sugars, such as the $\alpha$ oxoaldehydes methylglyoxal, glyoxal and 3-deoxyglucosone, react non-enzymatically with nucleophilic groups on proteins to form adducts [4]. Both reducing sugar adducts and $\alpha$ oxoaldehydes are irreversibly converted into AGE in a process accompanied by protein cross-linking. AGE have been implicated in the development of atherosclerosis by receptor and non-receptor-mediated mechanisms [5, 6], as well as in the development of endothelial dysfunction and diabetic microvascular complications [7, 8]. As plasma methylglyoxal levels can be elevated in individuals with diabetes (up to $0.5 \mathrm{mmol} / \mathrm{l}$ compared with approximately $100 \mu \mathrm{mol} / \mathrm{l}$ in unaffected individuals) [9], its involvement in AGE formation is a subject of considerable interest.

HDL are the smallest and most dense of all the plasma lipoproteins. Population studies have established that plasma HDL levels are inversely related to the risk of developing cardiovascular disease [10]. These anti-atherogenic properties are probably related to the participation of HDL in reverse cholesterol transport [11], as well as to their antioxidant, anti-inflammatory and anti-thrombotic properties [12-14]. HDL also improve endothelial function [15] and enhance endothelial repair [16].

There is mounting evidence to suggest that the antiatherogenic properties of HDL are decreased in patients with diabetes. For example, the ability of HDL from diabetic patients to export cholesterol from macrophages and act as a substrate for lecithin:cholesterol acyltransferase (LCAT), two key events in reverse cholesterol transport, is significantly reduced compared with that of HDL from normal persons [17, 18]. The anti-oxidant and antiinflammatory properties of HDL are also impaired in diabetic patients $[19,20]$.

We have recently shown that the apolipoprotein (Apo)AI in discoidal reconstituted HDL (rHDL) containing phosphatidylcholine and ApoA-I ([A-I]rHDL) becomes nonenzymatically glycated and cross-linked when it is incubated in vitro with 0.5 to $30 \mathrm{mmol} / 1$ methylglyoxal [21]. These modifications were accompanied by changes to the conformation of ApoA-I, the generation of epitopes that are recognised by anti-AGE antibodies and a reduction in the ability of ApoA-I to activate LCAT [21]. The present study was carried out to determine whether these detrimental structural and functional modifications could be prevented and/or reversed by agents with diverse mechanisms of action, such as the cross-link inhibitors aminoguanidine and pyridoxamine, the widely used insulin-sensitiser metformin or the cross-link breaker 4,5-dimethyl-3-phenacylthiozolium chloride (alagebrium).

The results show that aminoguanidine, alagebrium and, to a lesser extent, pyridoxamine and metformin protect ApoA-I from modification by methylglyoxal and conserve its ability to activate LCAT. However, none of these agents was able to reverse the adverse structural and functional changes that were mediated by methylglyoxal.

\section{Methods}

Isolation of ApoA-I HDL were ultracentrifugally isolated from pooled, autologously donated human plasma samples (Gribbles Pathology, Adelaide, SA, Australia). This was achieved with a single $16 \mathrm{~h}$ spin at a density of $1.07 \mathrm{~g} / \mathrm{ml}$ followed by two $26 \mathrm{~h}$ spins at $1.21 \mathrm{~g} / \mathrm{ml}$. All of the spins were carried out at $4^{\circ} \mathrm{C}$ in a Beckman $55.2 \mathrm{Ti}$ rotor (Beckman Coulter, Fullerton, CA, USA). The HDL were delipidated and ApoA-I was isolated from the resulting ApoHDL by anion exchange chromatography on a Q Sepharose Fast Flow column (GE Healthcare, Chalfont St Giles, UK) $[22,23]$. The ApoA-I appeared as a single band when subjected to SDS-PAGE and Coomassie staining.

Preparation of discoidal $r H D L$ Discoidal rHDL containing 1-palmitoyl-2-oleoyl phosphatidylcholine (POPC) (Avanti Polar Lipids, Alabaster, AL, USA), unesterified cholesterol (UC) (Sigma-Aldrich, St Louis, MO, USA), ApoA-I and a tracer amount of $\left[1 \alpha, 2 \alpha-{ }^{3} \mathrm{H}\right] \mathrm{UC}$ (GE Healthcare) (initial POPC:UC:A-I molar ratio 100:10:1) were prepared by the cholate dialysis method and dialysed against $6.5 \mathrm{mmol} / \mathrm{l} \mathrm{PBS}$, $\mathrm{pH} 7.4$, containing $0.006 \%$ (wt/vol.) $\mathrm{NaN}_{3}$ and $0.005 \%$ (wt/vol.) EDTA-Na $\mathrm{Na}_{2}$ before use [24].

Isolation of LCAT LCAT was isolated from pooled, human plasma as described [25] and appeared as a single band on a silver stained, homogeneous 20\% SDS-Phastgel (GE Healthcare). LCAT activity was assessed using discoidal (A-I)rHDL radiolabelled with a trace amount of $\left[1 \alpha, 2 \alpha-{ }^{3} \mathrm{H}\right]$ $\mathrm{UC}$ as the substrate [26]. The amount of cholesteryl ester generated by the LCAT preparations used in this study was 0.6 to $3.1 \mu \mathrm{mol}(\mathrm{ml} \mathrm{LCAT})^{-1} \mathrm{~h}^{-1}$.

Inhibition of methylglyoxal-mediated non-enzymatic glycation of ApoA-I in discoidal rHDL by aminoguanidine, pyridoxamine, metformin and alagebrium Discoidal (A-I)rHDL (final ApoA-I concentration $1 \mathrm{mg} / \mathrm{ml}$ ) were incubated for $24 \mathrm{~h}$ at 
$37^{\circ} \mathrm{C}$ in the absence or presence of $3 \mathrm{mmol} / \mathrm{l}$ methylglyoxal (Sigma-Aldrich) and aminoguanidine (Sigma-Aldrich), pyridoxamine, (Sigma-Aldrich), metformin (Sigma-Aldrich) or alagebrium (Alteon, Montvale, NJ, USA) (final concentrations $3 \mathrm{mmol} / 1$ in all cases). When the incubations were complete, the mixtures were dialysed against PBS. In additional experiments discoidal (A-I)rHDL were incubated in the absence or presence of $3 \mathrm{mmol} / \mathrm{l}$ methylglyoxal and 1.5 , 3 or $6 \mathrm{mmol} / \mathrm{l}$ aminoguanidine.

Reversal of methylglyoxal-mediated non-enzymatic glycation of ApoA-I in discoidal rHDL by aminoguanidine, pyridoxamine and alagebrium Discoidal (A-I)rHDL (final ApoA-I concentration $1 \mathrm{mg} / \mathrm{ml}$ ) were incubated at $37^{\circ} \mathrm{C}$ for $24 \mathrm{~h}$ with $3 \mathrm{mmol} / 1$ methylglyoxal. The incubation mixtures were dialysed against PBS to remove the unreacted methylglyoxal, then incubated for an additional $24 \mathrm{~h}$ at $37^{\circ} \mathrm{C}$ in the presence or absence of either aminoguanidine, pyridoxamine or alagebrium (final concentration $3 \mathrm{mmol} / \mathrm{l}$ in all cases). The samples were then dialysed again against PBS.

Assessment of ApoA-I arginine, lysine and tryptophan residue modification ApoA-I arginine, lysine and tryptophan residue modifications were assessed fluorometrically and expressed as a percentage of the value obtained for control samples incubated without methylglyoxal [27].

Agarose gel electrophoresis Discoidal (A-I)rHDL was were electrophoresed at $90 \mathrm{~V}$ for $45 \mathrm{~min}$ on $1 \%(\mathrm{wt} / \mathrm{vol}$.) agarose gels (Helena Laboratories, Mt. Waverly, VIC, Australia), fixed in methanol, stained with Coomasie Blue $\mathrm{R}$ and destained with a buffer containing $10 \%$ (vol./vol.) acetic acid and $12.5 \%$ (vol./vol.) isopropanol.

Electrophoretic mobilities were calculated as

mobility $=\frac{\text { migration distance }(\mu \mathrm{m}) / \operatorname{time}(\mathrm{s})}{\operatorname{voltage}(\mathrm{V}) / \text { length of } \operatorname{gel}(\mathrm{cm})}$

and corrected for isoelectric point $(\mathrm{p} I)$-dependent retardation effects [28]:

mobility $_{\text {corrected }}=\left(\right.$ mobility $\left._{\text {agarose }}-0.136\right) / 1.21$

Kinetic studies Discoidal (A-I)rHDL (final unlabelled UC concentration $0.5-14 \mu \mathrm{mol} / \mathrm{l})$ containing a tracer amount of $\left[1 \alpha, 2 \alpha-{ }^{3} \mathrm{H}\right] \mathrm{UC}$ were incubated at $37^{\circ} \mathrm{C}$ for $1 \mathrm{~h}$ in a shaking water bath with LCAT, fatty acid-free bovine serum albumin (final concentration $3.7 \mathrm{mg} / \mathrm{ml}$ ), $\beta$-mercaptoethanol (final concentration $5.3 \mathrm{mmol} / \mathrm{l}$ ) and Tris-buffered saline, (pH 7.4). The final volume of the incubation mixtures was $135 \mu \mathrm{l}$. Cholesterol esterification was determined as described [26]. The incubations were carried out in triplicate on three separate occasions. Michaelis-Menten constant $\left(K_{\mathrm{m}}\right)_{(\mathrm{app})}$ and the maximum rate of cholesterol esterification $\left(V_{\max }\right)$ were determined by non-linear regression analysis (GraphPad Prism Version 4.0b; GraphPad, San Diego, CA, USA) using the equation:

$V=\left(V_{\max } \times[\mathrm{UC}]\right) /\left(K_{\mathrm{m}(\mathrm{app})}+[\mathrm{UC}]\right)$

where $V$ is the rate of cholesterol esterification and $[\mathrm{UC}]$ is the concentration of unlabelled UC in the discoidal (A-I) rHDL. The results are expressed, as amount of cholesteryl ester formed, in $\mu \mathrm{mol}(\mathrm{ml} \mathrm{LCAT})^{-1} \mathrm{~h}^{-1}$.

Other techniques UC and phospholipid concentrations were measured enzymatically $[29,30]$. The bicinchoninic assay was used to determine ApoA-I concentrations using BSA as a standard [31]. All concentrations were determined in triplicate on an automatic analyser (Hitachi 902; Roche Diagnostics, Mannheim, Germany). Protein cross-linking was assessed by SDS-PAGE. Discoidal (A-I)rHDL diameters were determined by electrophoresis on $3 / 40 \%$ nondenaturing polyacrylamide gradient gels and staining with Coomassie Blue R [32]. Particle sizes were determined by reference to high molecular mass standards of known diameter.

Statistical analysis GraphPad Prism Version 4.0b for Macintosh (Graph-Pad) was used for all statistical analyses. One-way ANOVA with Tukey's multiple comparison posttest analysis was used to determine whether the ApoA-I lysine, arginine and tryptophan residues were modified significantly. Differences between cholesterol esterification rates were assessed by two-way ANOVA. In all cases significance was set at $p<0.05$.

\section{Results}

Effects of methylglyoxal, aminoguanidine, pyridoxamine, metformin and alagebrium on the physical properties of discoidal (A-I)rHDL The discoidal (A-I)rHDL (final POPC:UC:ApoA-I molar ratio 145:9:1) consisted of three major populations of particles $16.4,11.9$ and $9.9 \mathrm{~nm}$ in diameter (not shown). Incubation with $3 \mathrm{mmol} / \mathrm{l}$ methylglyoxal did not affect the overall stoichiometry of the discoidal (A-I)rHDL, but did decrease the particle diameters to 15.9, 11.6 and $9.3 \mathrm{~nm}$, respectively. This probably reflects changes to the conformation of ApoA-I [21]. Inclusion of aminoguanidine, pyridoxamine, metformin or alagebrium in the incubations of discoidal (A-I)rHDL with methylglyoxal did not alter the size or the stoichiometry of the particles (not shown). 
Inhibition of methylglyoxal-mediated non-enzymatic glycation of ApoA-I in discoidal rHDL by aminoguanidine, metformin, pyridoxamine and alagebrium We have shown previously that incubation of discoidal (A-I)rHDL with $0.5 \mathrm{mmol} / 1$ methylglyoxal, a concentration comparable to that reported in the plasma of diabetic patients, significantly reduces the ability of the particles to act as substrates for LCAT [9, 21]. However, the extent to which the ApoA-I arginine, lysine and tryptophan residues were modified in those studies was below the level of detection [21]. To ensure that modifications to ApoA-I could be detected in the present experiments, all of the incubations were carried out with $3 \mathrm{mmol} / \mathrm{l}$ methylglyoxal.

After $24 \mathrm{~h}$ of incubation with $3 \mathrm{mmol} / \mathrm{l}$ methylglyoxal, 59.4, 69.2 and 72.4\% of the ApoA-I lysine, arginine and tryptophan residues in the discoidal (A-I)rHDL respectively remained unmodified (Table 1). The ApoA-I arginine, lysine and tryptophan residues in the discoidal rHDL that were incubated with methylglyoxal as well as with aminoguanidine, pyridoxamine, metformin or alagebrium were modified to a lesser extent than those incubated with methylglyoxal alone. The arginine, lysine and tryptophan residues in the discoidal (A-I)rHDL that were incubated with methylglyoxal as well as aminoguanidine, pyridoxamine, metformin or alagebrium were, however, significantly modified compared with changes observed for discoidal (A-I)rHDL that had not been incubated with methylglyoxal. Modification of the lysine and tryptophan residues in the (A-I)rHDL that were incubated with methylglyoxal and alagebrium could not be determined because of the formation of a methylglyoxal-alagebrium complex, the fluorescence emission spectrum of which overlapped that of the samples (Table 1).
An additional study was carried out to determine whether aminoguanidine was able to inhibit methylglyoxal-mediated modifications to ApoA-I lysine, arginine and tryptophan residues in discoidal (A-I)rHDL in a concentration-dependent manner (Fig. 1). Modification of the ApoA-I lysine and tryptophan residues was inhibited to an equal extent by 1.5 and $3 \mathrm{mmol} / \mathrm{l}$ aminoguanidine. When $6 \mathrm{mmol} / \mathrm{l}$ aminoguanidine was included in the incubations, the methylglyoxalmediated modifications to the ApoA-I arginine, lysine and tryptophan residues were no longer apparent.

As judged by agarose gel electrophoresis, incubation with methylglyoxal increased the negative charge of the discoidal (A-I)rHDL surface (Table 1). The reduction in rHDL surface charge was not as great when aminoguanidine, alagebrium and, to a lesser extent, pyridoxamine and metformin were included in the incubations.

Ability of aminoguanidine, metformin, pyridoxamine and alagebrium to inhibit methylglyoxal-mediated structural modifications to ApoA-I in discoidal rHDL When discoidal (A-I)rHDL were incubated in the absence of methylglyoxal, then subjected to SDS-PAGE, the ApoA-I appeared as a single band (Fig. 2). When methylglyoxal was included in the incubation, the molecular mass of the ApoA-I increased. This is consistent with cross-linking of the ApoA-I. Inclusion of either $3 \mathrm{mmol} / \mathrm{l}$ aminoguanidine or alagebrium in the incubations markedly attenuated ApoA-I cross-linking. Inclusion of either 1.5 or $6 \mathrm{mmol} / \mathrm{l}$ aminoguanidine in the incubations inhibited ApoA-I cross-linking to the same extent as $3 \mathrm{mmol} / \mathrm{l}$ aminoguanidine (not shown). Pyridoxamine and metformin inhibited the methylglyoxal-mediated ApoA-I cross-linking less effectively than either aminoguanidine or alagebrium.

Table 1 Characterisation of discoidal (A-I)rHDL after incubation with methylglyoxal and aminoguanidine, pyridoxamine, metformin or alagebrium

\begin{tabular}{lllll}
\hline \multirow{2}{*}{ Additions } & \multicolumn{2}{l}{ Unmodified residues (\%) } & \multirow{2}{*}{ Electrophoretic mobility } \\
\cline { 2 - 5 } & Arginine & Lysine & Tryptophan & \\
\hline No methylglyoxal & 100 & 100 & 100 & -0.38 \\
Methylglyoxal & $59.4 \pm 2.6^{\mathrm{a}}$ & $69.2 \pm 0.3^{\mathrm{a}}$ & $72.4 \pm 0.6^{\mathrm{a}}$ & -0.55 \\
Methylglyoxal + aminoguanidine & $89.9 \pm 2.7^{\mathrm{a}, \mathrm{c}}$ & $89.3 \pm 0.2^{\mathrm{a}, \mathrm{c}}$ & $88.8 \pm 0.9^{\mathrm{a}, \mathrm{c}}$ & -0.41 \\
Methylglyoxal + pyridoxamine & $73.0 \pm 5.1^{\mathrm{a}, \mathrm{c}}$ & $83.4 \pm 0.8^{\mathrm{a}, \mathrm{c}}$ & $86.0 \pm 0.8^{\mathrm{a}, \mathrm{c}}$ & -0.48 \\
Methylglyoxal + metformin & $71.1 \pm 0.2^{\mathrm{a}, \mathrm{b}}$ & $80.1 \pm 0.8^{\mathrm{a}, \mathrm{c}}$ & $80.8 \pm 0.5^{\mathrm{a}, \mathrm{c}}$ & -0.50 \\
Methylglyoxal + alagebrium & $70.8 \pm 0.8^{\mathrm{a}, \mathrm{c}}$ & nd & nd & -0.43
\end{tabular}

Discoidal (A-I)rHDL (final concentration $1 \mathrm{mg} / \mathrm{ml} \mathrm{ApoA-I}$ ) were incubated at $37^{\circ} \mathrm{C}$ for $24 \mathrm{~h}$ with methylglyoxal (final concentration $3 \mathrm{mmol} / \mathrm{l}$ ) in the absence or presence of $3 \mathrm{mmol} / \mathrm{l}$ aminoguanidine, pyridoxamine, metformin or alagebrium. The final volume of the incubation mixtures was $4 \mathrm{ml}$. Modification of arginine, lysine and tryptophan residues was quantified by fluorescence. Electrophoretic mobilities $\left(\mu \mathrm{m} \mathrm{s} / / \mathrm{cm} \mathrm{V}^{-1}\right)$ were determined as described in the Methods section

${ }^{\mathrm{a}} p<0.001$ compared with unmodified discoidal (A-I)rHDL; ${ }^{\mathrm{b}} p<0.05,{ }^{\mathrm{c}} p<0.001$ compared with discoidal (A-I)rHDL incubated with 3 mmol/1 methylglyoxal

nd, not determined 


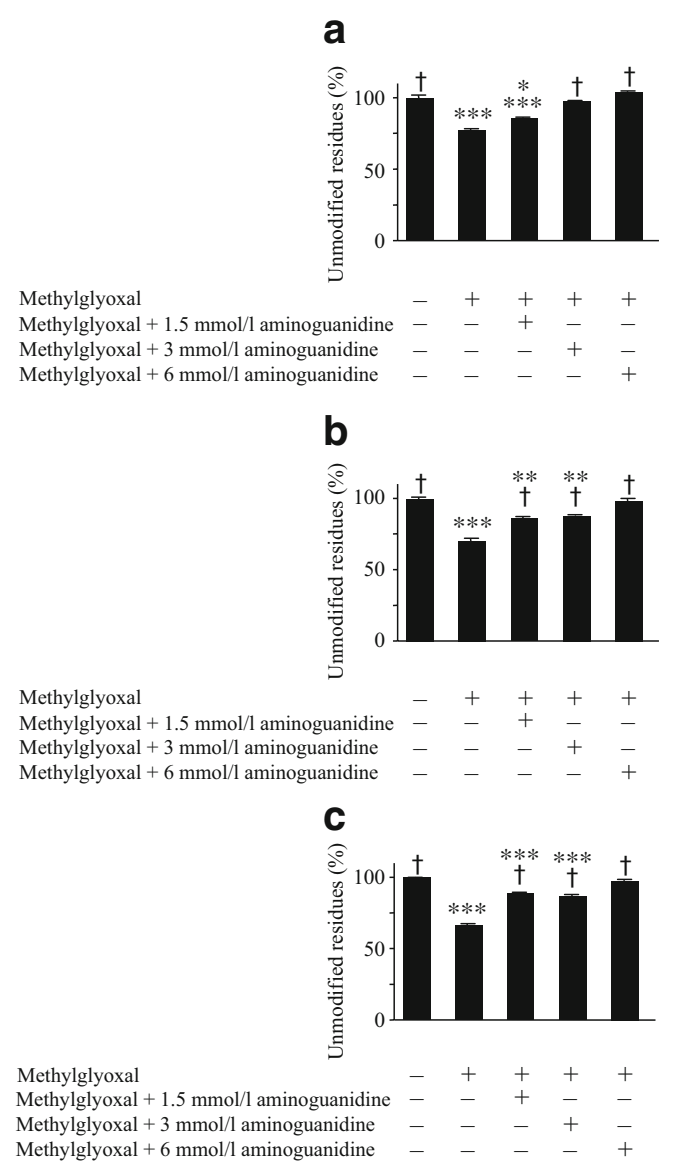

Fig. 1 Modification of ApoA-I amino acid residues in discoidal rHDL by incubation with methylglyoxal $(3 \mathrm{mmol} / \mathrm{l})$ and varying concentrations of aminoguanidine. Discoidal (A-I)rHDL (final ApoAI concentration $1 \mathrm{mg} / \mathrm{ml}$ ) were incubated for $24 \mathrm{~h}$ at $37^{\circ} \mathrm{C}$ in the absence or presence of methylglyoxal and aminoguanidine as indicated. The final volume of the incubation mixtures was $1.7 \mathrm{ml}$. Modification of arginine (a), lysine (b) and tryptophan (c) residues was quantified by fluorescence. ${ }^{*} p<0.05$ versus (A-I)rHDL incubated in the presence of methylglyoxal; $* * p<0.01$ and $* * * p<0.001$ versus (A-I)rHDL incubated in the absence of methylglyoxal; $\uparrow p<0.001$ versus (A-I)rHDL incubated in the presence of methylglyoxal

Effects of aminoguanidine, metformin, pyridoxamine and alagebrium on HDL function As the results in Figs 1 and 2 and Table 1 are consistent with the attenuation of methylglyoxal-mediated structural changes in discoidal (AI)rHDL by aminoguanidine, metformin, pyridoxamine and alagebrium, it was important to know whether these agents also improved the ability of the particles to act as substrates for LCAT. This was achieved by determining whether discoidal (A-I)rHDL incubated with methylglyoxal as well as aminoguanidine, pyridoxamine, metformin or alagebrium acted as a better substrate for LCAT than discoidal (A-I) rHDL that had been incubated with methylglyoxal alone. Compared with discoidal (A-I)rHDL incubated in the absence of methylglyoxal, the rate of cholesterol esterification in the rHDL that had been incubated for $24 \mathrm{~h}$ with

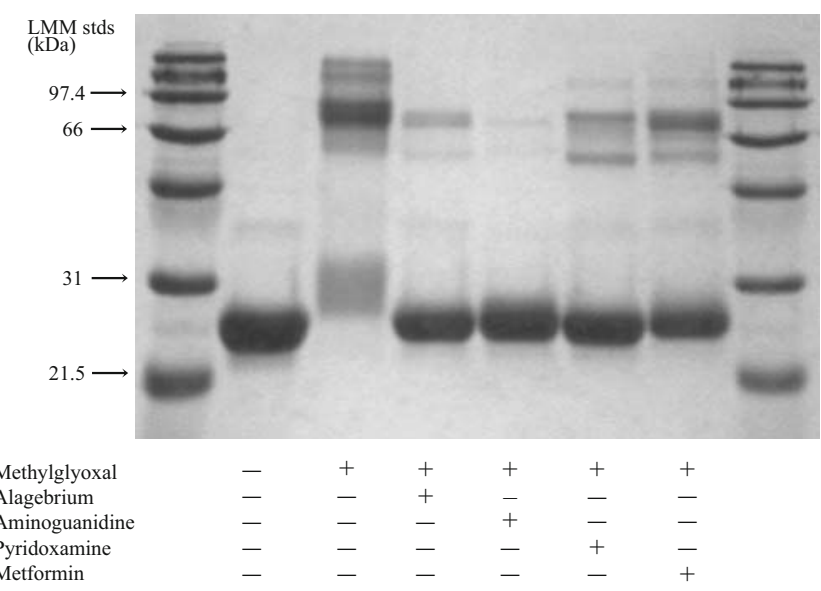

Fig. 2 Effects of methylglyoxal, alagebrium, aminoguanidine, pyridoxamine and metformin on ApoA-I cross-linking in discoidal rHDL. Discoidal (A-I)rHDL (final ApoA-I concentration $1 \mathrm{mg} / \mathrm{ml}$ ) were incubated for $24 \mathrm{~h}$ at $37^{\circ} \mathrm{C}$ in the absence or presence of methylglyoxal and either aminoguanidine, pyridoxamine, metformin or alagebrium (final concentration $3 \mathrm{mmol} / \mathrm{l}$ for all). When the incubations were complete, the mixtures were dialysed against PBS and subjected to SDS-PAGE. A scan of a gel stained with Coomassie Blue $\mathrm{R}$ is shown. LMM stds, low molecular mass standards

methylglyoxal was minimal (Fig. 3). When the (A-I)rHDL that had been incubated with methylglyoxal and either aminoguanidine, alagebrium, pyridoxamine or metformin were incubated with LCAT, the rate of cholesterol esterification was enhanced compared with that observed for (A-I) rHDL incubated with methylglyoxal alone. The greatest enhancement of cholesterol esterification was observed in

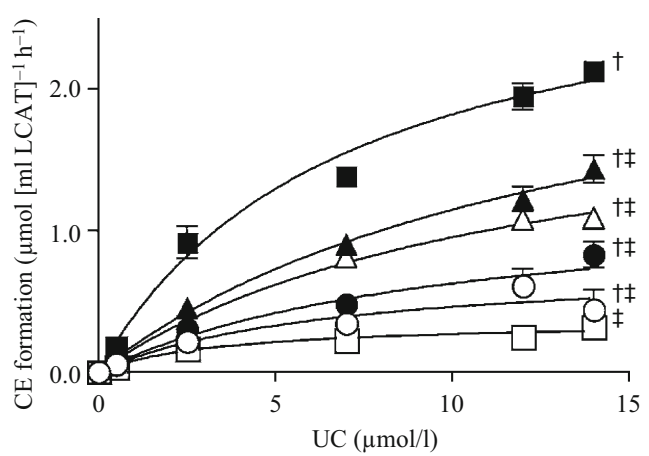

Fig. 3 LCAT-mediated cholesterol esterification in discoidal (A-I) rHDL incubated with methylglyoxal and either alagebrium, aminoguanidine, pyridoxamine or metformin. Discoidal (A-I)rHDL were incubated at $37^{\circ} \mathrm{C}$ for $24 \mathrm{~h}$ in the absence (black squares) or presence (white squares) of $3 \mathrm{mmol} / \mathrm{l}$ methylglyoxal and either aminoguanidine (black triangles), pyridoxamine (black circles), metformin (white circles) or alagebrium (white triangles) (final concentration $3 \mathrm{mmol} / \mathrm{l}$ in all cases). The rHDL were then incubated at $37^{\circ} \mathrm{C}$ for $1 \mathrm{~h}$ with $5 \mu \mathrm{l}$ of a $1 / 50$ dilution of an LCAT preparation and the rate of cholesterol esterification was determined. Amount of cholesteryl ester (CE) generated by LCAT preparation: $3.1 \mu \mathrm{mol}(\mathrm{ml} \mathrm{LCAT})^{-1} \mathrm{~h}^{-1}$ as described in the Methods section. ${ }^{\dagger} p<0.0001$ versus (A-I)rHDL incubated in the presence of methylglyoxal; ${ }^{\star} p<0.0001$ versus (A-I)rHDL incubated in the absence of methylglyoxal 
the incubations that contained aminoguanidine. Irrespective of whether a cross-link breaker, a glycation inhibitor or an insulin sensitiser was included in the incubations, the rate of cholesterol esterification was lower than that observed for discoidal (A-I)rHDL incubated in the absence of methylglyoxal.

Kinetic parameters for the LCAT reaction are shown in Table 2. As previously reported [21], the rate of cholesterol esterification was very low in the discoidal (A-I)rHDL incubated with methylglyoxal alone. When (A-I)rHDL were incubated with methylglyoxal and either aminoguanidine, alagebrium, pyridoxamine or metformin, the $V_{\max }$ was markedly increased compared with that observed for (A-I)rHDL incubated with methylglyoxal alone. However, the rate of cholesterol esterification in these samples was still less than that of discoidal (A-I)rHDL that had not been incubated with methylglyoxal. As there were only small variations in the $K_{\mathrm{m}(\mathrm{app})}$ values, it follows that incubation with methylglyoxal, aminoguanidine, alagebrium, pyridoxamine and metformin did not affect the affinity of LCAT for (A-I)rHDL.

Given that aminoguanidine enhanced the reactivity of discoidal (A-I)rHDL with LCAT more effectively than any of the other agents, a further experiment was carried out to determine whether this improvement was concentrationdependent. This was achieved by incubating discoidal (A-I) rHDL with $3 \mathrm{mmol} / 1$ methylglyoxal and either $1.5,3$ or $6 \mathrm{mmol} / \mathrm{l}$ aminoguanidine (Table 3). The results of this experiment showed that the rate of cholesterol esterification increased when incubations of (A-I)rHDL and methylglyoxal were supplemented with either 1.5 or $3 \mathrm{mmol} / 1$ aminoguanidine. When (A-I)rHDL were incubated with methylglyoxal and $6 \mathrm{mmol} / \mathrm{l}$ aminoguanidine, the $V_{\max }$ for cholesterol esterification was indistinguishable from that observed for (A-I)rHDL not incubated with methylglyoxal. This is consistent with the observation in Fig. 1, which shows that ApoA-I lysine, arginine and tryptophan residues were not modified when (A-I)rHDL were incubated with methylglyoxal and $6 \mathrm{mmol} / \mathrm{l}$ aminoguanidine.

Ability of aminoguanidine, alagebrium and pyridoxamine to reverse methylglyoxal-mediated non-enzymatic glycation of ApoA-I in discoidal rHDL The aim of this study was to determine whether aminoguanidine, pyridoxamine or alagebrium could reverse the deleterious structural and functional effects that methylglyoxal exerts on discoidal (A-I)rHDL. This was achieved by incubating discoidal (A-I)rHDL with methylglyoxal, removing the unreacted methylglyoxal from the incubation mixtures, then further incubating the rHDL with aminoguanidine, pyridoxamine or alagebrium. None of these compounds were able to reverse the methylglyoxal-mediated modification of ApoA-I arginine, lysine and tryptophan residues (Table 4) or reduce ApoA-I crosslinking (not shown). Aminoguanidine, pyridoxamine and alagebrium were also unable to restore the ability of methylglyoxal-modified discoidal (A-I)rHDL to act as a substrate for LCAT (not shown).

\section{Discussion}

This study shows for the first time that the deleterious structural and functional changes that occur when ApoA-I is modified by the reactive $\alpha$-oxoaldehyde, methylglyoxal, can be prevented, but not reversed by the cross-link breaker alagebrium, the glycation inhibitors aminoguanidine and pyridoxamine, and the insulin sensitiser metformin. In addition to preventing these methylglyoxal-mediated modifications to (A-I)rHDL, our results also show that aminoguanidine, pyridoxamine, metformin and alagebrium conserve the ability of ApoA-I to activate LCAT, the key event of the second step in the potentially anti-atherogenic reverse cholesterol transport pathway.

Table 2 Effects of aminoguanidine, pyridoxamine, metformin and alagebrium on the kinetics of the LCAT reaction in discoidal rHDL containing non-enzymatically glycated ApoA-I

\begin{tabular}{|c|c|c|c|c|c|c|}
\hline & \multicolumn{6}{|c|}{ rHDL with(+)/without(-) } \\
\hline & $-\mathrm{MG}$ & $+\mathrm{MG}$ & $+\mathrm{MG}+$ aminoguanidine & $+\mathrm{MG}+$ pyridoxamine & $+\mathrm{MG}+$ metformin & $+\mathrm{MG}+$ alagebrium \\
\hline$V_{\max }^{\mathrm{a}}$ & $3.07 \pm 0.28$ & $0.37 \pm 0.05$ & $2.72 \pm 0.44$ & $1.26 \pm 0.28$ & $0.77 \pm 0.28$ & $2.10 \pm 0.29$ \\
\hline$K_{m(\text { app })}^{b}$ & $6.88 \pm 1.51$ & $3.60 \pm 1.47$ & $13.76 \pm 3.91$ & $10.09 \pm 4.47$ & $6.85 \pm 5.92$ & $12.08 \pm 3.10$ \\
\hline
\end{tabular}

Discoidal (A-I)rHDL (final concentration ApoA-I $1 \mathrm{mg} / \mathrm{ml}$ ) were incubated at $37^{\circ} \mathrm{C}$ for $24 \mathrm{~h}$ with methylglyoxal (final concentration $3 \mathrm{mmol} / \mathrm{l}$ ) in the presence or absence of aminoguanidine, pyridoxamine, metformin and alagebrium $(3 \mathrm{mmol} / 1 \mathrm{in}$ each case). When the incubations were complete, rHDL were dialysed against $3 \times 11$ PBS The (A-I)rHDL (final UC concentration $0.5-14 \mu \mathrm{mol} / \mathrm{l}$ ) were then incubated for $1 \mathrm{~h}$ with $5 \mu \mathrm{l}$ of a 1/50 dilution of a preparation of LCAT that generated $3.1 \mu \mathrm{mol}$ cholesteryl ester $(\mathrm{ml} \mathrm{LCAT})^{-1} \mathrm{~h}^{-1}$

Kinetic parameters were derived by non-linear regression analysis of the rate of cholesterol esterification versus the concentration of substrate

${ }^{\text {a }}$ For formation of cholesteryl ester in $\mu \mathrm{mol}(\mathrm{ml} \mathrm{LCAT})^{-1} \mathrm{~h}^{-1}$

${ }^{\mathrm{b}}$ In $\mu \mathrm{mol} \mathrm{UC}$

MG, methylglyoxal 
Table 3 Effects of varying concentrations of aminoguanidine on the kinetics of the LCAT reaction in discoidal rHDL containing nonenzymatically glycated ApoA-I

\begin{tabular}{lll}
\hline Incubation conditions & $V_{\max }^{\mathrm{a}}$ & $K_{\mathrm{m}(\mathrm{app})}^{\mathrm{b}}$ \\
\hline $\begin{array}{l}\text { No methylglyoxal } \\
\text { Methylglyoxal }\end{array}$ & $1.25 \pm 0.19$ & $20.64 \pm 6.61$ \\
$\begin{array}{l}\text { Methylglyoxal }+1.5 \mathrm{mmol} / 1 \\
\quad \text { aminoguanidine }\end{array}$ & $0.76 \pm 0.11$ & $13.31 \pm 3.7$ \\
$\begin{array}{l}\text { Methylglyoxal }+3 \mathrm{mmol} / 1 \\
\quad \text { aminoguanidine }\end{array}$ & $0.82 \pm 0.08$ & $31.43 \pm 4.82$ \\
$\begin{array}{l}\text { Methylglyoxal }+6 \mathrm{mmol} / 1 \\
\quad \text { aminoguanidine }\end{array}$ & $1.29 \pm 0.11$ & $20.3 \pm 3.73$ \\
\hline
\end{tabular}

Discoidal (A-I)rHDL (final concentration ApoA-I $1 \mathrm{mg} / \mathrm{ml}$ ) were incubated at $37^{\circ} \mathrm{C}$ for $24 \mathrm{~h}$ with methylglyoxal (final concentration $3 \mathrm{mmol} / \mathrm{l})$ in presence or absence of aminoguanidine $(1.5,3$ or $6 \mathrm{mmol} / \mathrm{l})$. When the incubations were complete the mixtures were dialysed against PBS and incubated as described in Table 2 with $5 \mu 1$ of a 1/10 dilution of an LCAT preparation that generated $0.6 \mu \mathrm{mol}$ cholesteryl ester (ml LCAT) $)^{-1} \mathrm{~h}^{-1}$

Kinetic parameters were derived by non-linear regression analysis of the rate of cholesterol esterification versus the concentration of substrate

${ }^{\mathrm{a}}$ For formation of cholesteryl ester in $\mu \mathrm{mol}(\mathrm{ml} \mathrm{LCAT})^{-1} \mathrm{~h}^{-1}$

${ }^{\mathrm{b}}$ In $\mu \mathrm{mol} \mathrm{UC}$

Although it is well established that aminoguanidine inhibits the non-enzymatic glycation of various plasma proteins and their conversion into AGE [33], little is known about its effects on methylglyoxal-mediated modifications of ApoA-I or on HDL function. Aminoguanidine probably reduces the ability of methylglyoxal to modify ApoA-I by mechanisms that are similar to those reported for other proteins. This may involve the scavenging and inactivation of methylglyoxal in processes that decrease its availability. Aminoguanidine may also directly decrease AGE formation by interacting irreversibly with Amadori intermediates [34] or by chelating trace amounts of transition metals that are required to catalyse their production [35].
Even though the mechanisms whereby metformin prevents the non-enzymatic glycation of ApoA-I are not known, its structural similarity to aminoguanidine suggests that it probably attenuates the non-enzymatic glycation of ApoA-I by similar pathways [36, 37]. However, the current results show that aminoguanidine and metformin are not equally effective in reducing methylglyoxal-mediated modifications to ApoA-I. While the underlying reasons for this difference are not entirely clear, they may be explained by a recent observation showing that aminoguanidine scavenges methylglyoxal more effectively than metformin [38]. It is also possible that structural differences between the two compounds may change the kinetics of their interactions with either methylglyoxal or with the initial adducts that are formed when methylglyoxal interacts with ApoA-I.

The possibility that metformin and aminoguanidine may prevent methylglyoxal from modifying ApoA-I by mechanisms that are distinct from each other cannot be dismissed. This is certainly the case for pyridoxamine, which reportedly forms complexes with transition metal ions, thereby preventing metal-ion-catalysed oxidation of postAmadori products and their subsequent conversion into AGE [39]. Pyridoxamine has also been reported to scavenge the carbonyl intermediates generated during AGE formation [40]. These processes inhibit AGE formation in vivo very effectively, at least in studies of diabetic rats, where significant delays in the onset of retinopathy and nephropathy have been reported [41, 42].

One of the main aims of the present study was to determine whether the ability of aminoguanidine, pyridoxamine, metformin and alagebrium to prevent methylglyoxal modifications to ApoA-I also has a positive influence on HDL function. The present results show that this is the case, with all of these agents preserving the ability of HDL to act as a substrate for LCAT and thus maintain the second step of the reverse cholesterol transport pathway. This complements the findings of other investigators, who have

Table 4 Reversal of methylglyoxal-mediated ApoA-I modifications in discoidal rHDL by aminoguanidine, pyridoxamine and alagebrium

\begin{tabular}{|c|c|c|c|}
\hline \multirow[t]{2}{*}{ Additions } & \multicolumn{3}{|c|}{ Unmodified residues $(\%)$} \\
\hline & Arginine & Lysine & Tryptophan \\
\hline rHDL - methylglyoxal & 100 & 100 & 100 \\
\hline rHDL + methylglyoxal & $59.4 \pm 2.6$ & $69.2 \pm 0.3$ & $72.4 \pm 0.6$ \\
\hline rHDL + methylglyoxal + aminoguanidine & $66.4 \pm 2.9$ & $67.1 \pm 1.7$ & $69.7 \pm 0.1$ \\
\hline rHDL + methylglyoxal + pyridoxamine & $59.3 \pm 2.5$ & $71.2 \pm 1.1$ & $73.3 \pm 1.0$ \\
\hline rHDL + methylglyoxal + alagebrium & $66.4 \pm 2.9$ & nd & nd \\
\hline
\end{tabular}

Discoidal (A-I)rHDL (final concentration $1 \mathrm{mg} / \mathrm{ml}$ ApoA-I) were incubated at $37^{\circ} \mathrm{C}$ for $24 \mathrm{~h}$ with $3 \mathrm{mmol} / 1$ methylglyoxal, dialysed against PBS, then incubated for an additional $24 \mathrm{~h}$ at $37^{\circ} \mathrm{C}$ in the absence or presence of aminoguanidine, pyridoxamine or alagebrium ( $3 \mathrm{mmol} / 1$ for all). The final volume of the incubation mixtures was $4 \mathrm{ml}$. Modification of arginine, lysine and tryptophan residues was quantified by fluorescence as described in the Methods section nd, not determined 
found that aminoguanidine and metformin both enhance the first step of the reverse cholesterol pathway by increasing the efflux of cholesterol to $\mathrm{HDL}_{3}$ from ldlA7 cells stably transfected with scavenger receptor-B1. As part of the same study, aminoguanidine and metformin were also shown to enhance the export of cholesterol from cholesterol-loaded mouse peritoneal macrophages to $\mathrm{HDL}_{3}$ [43]. Hoang et al. have more recently reported that the reduced ability of AGE-modified ApoA-I to export cholesterol from monocytes, macrophages and HeLa cells transfected with ABCA1 is alleviated by aminoguanidine and alagebrium [44]. The ability of metformin and aminoguanidine to inhibit the glycation of LDL by methylglyoxal and glycoaldehyde also reduces the capacity of the lipoproteins to lipid-load macrophages [45].

When all of the above observations are considered together, it follows that aminoguanidine, metformin and alagebrium are likely to be beneficial in terms of reducing cardiovascular risk. Metformin is a well tolerated and widely used oral hypoglycaemic agent with a proven ability to reduce cardiovascular risk, while alagebrium decreases atherosclerotic lesion size in diabetic, ApoE-deficient mice [46]. Alagebrium has also been shown to improve endothelial function and reduce arterial stiffness in nondiabetic, hypertensive human patients in Phase 2a clinical trials [47]. Of particular importance was the absence of adverse effects in the participants in the latter studies. Thus, the current results indicate that, in addition to improving endothelial function and hyperglycaemia, metformin and alagebrium may also have beneficial effects on HDL function in diabetic patients. While this may also be the case for aminoguanidine, numerous adverse side effects have been reported for this agent in humans and animals $[48,49]$.

The present results with alagebrium are of particular interest. While several investigators have reported that alagebrium reduces collagen cross-linking in diabetic animals by cleaving the bonds between adjacent carbonyl groups of cross-linked proteins [50], other investigators have not been able to confirm these findings [51, 52]. We were also unable to demonstrate that alagebrium reverses the methylglyoxal-mediated cross-linking of ApoA-I. While it could be argued that the outcome of this experiment might have been positive if the incubation with alagebrium had been extended beyond $24 \mathrm{~h}$, the physiological relevance of this result would be questionable given that the half-life of ApoA-I in normal human plasma can be as low as $15 \mathrm{~h} \mathrm{[53]} \mathrm{and} \mathrm{possibly} \mathrm{even} \mathrm{lower} \mathrm{in} \mathrm{patients} \mathrm{with}$ diabetes [54]. Our results, however, do show that alagebrium can prevent methylglyoxal-mediated modifications to ApoA-I. This is presumably a reflection of it acting as a scavenger of methylglyoxal or of the adducts that are formed when methylglyoxal interacts with amino acid residues on ApoA-I, as outlined above for aminoguanidine and pyridoxamine.

In conclusion, the present results provide new and compelling evidence to suggest that glycation inhibitors, cross-link breakers and insulin sensitisers are potentially important for maintaining HDL function and reducing cardiovascular risk by preserving the reverse cholesterol transport pathway. It remains to be determined whether these agents are also of benefit in enhancing any of the other mechanisms that are responsible for the cardioprotective properties of HDL.

Acknowledgements This research was supported by the National Health and Medical Research Council of Australia Grant 222722 to K. A. Rye and P. J. Barter, and the Diabetes Australia Research Trust (to M. J. Davies). The authors also thank Alteon (Montvale, NJ, USA) for the supply of alagebrium.

Duality of interest The authors declare that there is no duality of interest associated with this manuscript.

\section{References}

1. Nathan DM, Cleary PA, Backlund JY et al (2005) Intensive diabetes treatment and cardiovascular disease in patients with type 1 diabetes. N Engl J Med 353:2643-2653

2. Kilhovd BK, Berg TJ, Birkeland KI, Thorsby P, Hanssen KF (1999) Serum levels of advanced glycation end products are increased in patients with type 2 diabetes and coronary heart disease. Diabetes Care 22:1543-1548

3. Schiel R, Franke S, Appel T et al (2004) Improvement of the quality of diabetes control and decrease in the concentrations of AGE-products in patients with type 1 and insulin-treated type 2 diabetes mellitus: results from a 10 year-prospective, populationbased survey on the quality of diabetes care in Germany (JEVIN). Eur J Med Res 9:391-399

4. Thorpe SR, Baynes JW (2003) Maillard reaction products in tissue proteins: new products and new perspectives. Amino Acids 25:275-281

5. Meng J, Sakata N, Takebayashi S et al (1998) Glycoxidation in aortic collagen from STZ-induced diabetic rats and its relevance to vascular damage. Atherosclerosis 136:355-365

6. Schmidt AM, Hori O, Chen JX et al (1995) Advanced glycation endproducts interacting with their endothelial receptor induce expression of vascular cell adhesion molecule-1 (VCAM-1) in cultured human endothelial cells and in mice. A potential mechanism for the accelerated vasculopathy of diabetes. J Clin Invest 96:1395-1403

7. Hammes HP, Alt A, Niwa T et al (1999) Differential accumulation of advanced glycation end products in the course of diabetic retinopathy. Diabetologia 42:728-736

8. Makita Z, Radoff S, Rayfield EJ et al (1991) Advanced glycosylation end products in patients with diabetic nephropathy. N Engl J Med 325:836-842

9. Lapolla A, Flamini R, Dalla Vedova A et al (2003) Glyoxal and methylglyoxal levels in diabetic patients: quantitative determina- 
tion by a new GC/MS method. Clin Chem Lab Med 41:11661173

10. Gordon T, Castelli WP, Hjortland MC, Kannel WB, Dawber TR (1977) High density lipoprotein as a protective factor against coronary heart disease. The Framingham Study. Am J Med 62: $707-714$

11. Lewis GF, Rader DJ (2005) New insights into the regulation of HDL metabolism and reverse cholesterol transport. Circ Res 96:1221-1232

12. Kontush A, Chantepie S, Chapman MJ (2003) Small, dense HDL particles exert potent protection of atherogenic LDL against oxidative stress. Arterioscler Thromb Vasc Biol 23:1881-1888

13. Nicholls SJ, Dusting GJ, Cutri B et al (2005) Reconstituted highdensity lipoproteins inhibit the acute pro-oxidant and proinflammatory vascular changes induced by a periarterial collar in normocholesterolemic rabbits. Circulation 111:1543-1550

14. Mineo C, Deguchi H, Griffin JH, Shaul PW (2006) Endothelial and antithrombotic actions of HDL. Circ Res 98:1352-1364

15. Spieker LE, Sudano I, Hurlimann D et al (2002) High-density lipoprotein restores endothelial function in hypercholesterolemic men. Circulation 105:1399-1402

16. Tso C, Martinic G, Fan WH, Rogers C, Rye KA, Barter PJ (2006) High-density lipoproteins enhance progenitor-mediated endothelium repair in mice. Arterioscler Thromb Vasc Biol 26:1144-1149

17. Passarelli M, Tang C, McDonald TO et al (2005) Advanced glycation end product precursors impair ABCA1-dependent cholesterol removal from cells. Diabetes 54:2198-2205

18. Ohta T, Nishiyama S, Nakamura T, Saku K, Maung KK, Matsuda I (1998) Predominance of large low density lipoprotein particles and lower fractional esterification rate of cholesterol in high density lipoprotein in children with insulin-dependent diabetes mellitus. Eur J Pediatr 157:276-281

19. Nobecourt E, Jacqueminet S, Hansel B et al (2005) Defective antioxidative activity of small dense HDL 3 particles in type 2 diabetes: relationship to elevated oxidative stress and hyperglycaemia. Diabetologia 48:529-538

20. Rohrer L, Hersberger M, von Eckardstein A (2004) High density lipoproteins in the intersection of diabetes mellitus, inflammation and cardiovascular disease. Curr Opin Lipidol 15:269-278

21. Nobecourt E, Davies MJ, Brown BE et al (2007) The impact of glycation on apolipoprotein A-I structure and its ability to activate lecithin:cholesterol acyltransferase. Diabetologia 50:643-653

22. Osborne JC Jr (1986) Delipidation of plasma lipoproteins. Methods Enzymol 128:213-222

23. Weisweiler P (1987) Isolation and quantitation of apolipoproteins A-I and A-II from human high-density lipoproteins by fast-protein liquid chromatography. Clin Chim Acta 169:249-254

24. Matz CE, Jonas A (1982) Micellar complexes of human apolipoprotein A-I with phosphatidylcholines and cholesterol prepared from cholate-lipid dispersions. J Biol Chem 257:4535-4540

25. Rye KA, Garrety KH, Barter PJ (1993) Preparation and characterization of spheroidal, reconstituted high-density lipoproteins with apolipoprotein A-I only or with apolipoprotein A-I and A-II. Biochim Biophys Acta 1167:316-325

26. Piran U, Morin RJ (1979) A rapid radioassay procedure for plasma lecithin-cholesterol acyltransferase. J Lipid Res 20:1040-1043

27. Knott HM, Brown BE, Davies MJ, Dean RT (2003) Glycation and glycoxidation of low-density lipoproteins by glucose and lowmolecular mass aldehydes. Formation of modified and oxidized particles. Eur J Biochem 270:3572-3582

28. Sparks DL, Phillips MC (1992) Quantitative measurement of lipoprotein surface charge by agarose gel electrophoresis. J Lipid Res 33:123-130

29. Stahler F, Gruber W, Stinshoff K, Roschlau P (1977) A practical enzymatic cholesterol determination. [Article in German] Med Lab (Stuttg) 30:29-37
30. Takayama M, Itoh S, Nagasaki T, Tanimizu I (1977) A new enzymatic method for determination of serum choline-containing phospholipids. Clin Chim Acta 79:93-98

31. Smith PK, Krohn RI, Hermanson GT et al (1985) Measurement of protein using bicinchoninic acid. Anal Biochem 150:76-85

32. Rainwater DL, Andres DW, Ford AL, Lowe F, Blanche PJ, Krauss RM (1992) Production of polyacrylamide gradient gels for the electrophoretic resolution of lipoproteins. J Lipid Res 33:18761881

33. Brownlee M, Vlassara H, Kooney A, Ulrich P, Cerami A (1986) Aminoguanidine prevents diabetes-induced arterial wall protein cross-linking. Science 232:1629-1632

34. Khatami M, Suldan Z, David I, Li W, Rockey JH (1988) Inhibitory effects of pyridoxal phosphate, ascorbate and aminoguanidine on nonenzymatic glycosylation. Life Sci 43:1725-1731

35. Price DL, Rhett PM, Thorpe SR, Baynes JW (2001) Chelating activity of advanced glycation end-product inhibitors. J Biol Chem 276:48967-48972

36. Rahbar S, Natarajan R, Yerneni K, Scott S, Gonzales N, Nadler JL (2000) Evidence that pioglitazone, metformin and pentoxifylline are inhibitors of glycation. Clin Chim Acta 301:65-77

37. Ruggiero-Lopez D, Lecomte M, Moinet G, Patereau G, Lagarde M, Wiernsperger N (1999) Reaction of metformin with dicarbonyl compounds. Possible implication in the inhibition of advanced glycation end product formation. Biochem Pharmacol 58:1765-1773

38. Battah S, Ahmed N, Thornalley PJ (2002) Kinetics and mechanism of the reaction of metformin with methylglyoxal. Int Congr Ser 1245:355-356

39. Voziyan PA, Khalifah RG, Thibaudeau C et al (2003) Modification of proteins in vitro by physiological levels of glucose: pyridoxamine inhibits conversion of Amadori intermediate to advanced glycation end-products through binding of redox metal ions. J Biol Chem 278:46616-46624

40. Voziyan PA, Metz TO, Baynes JW, Hudson BG (2002) A postAmadori inhibitor pyridoxamine also inhibits chemical modification of proteins by scavenging carbonyl intermediates of carbohydrate and lipid degradation. J Biol Chem 277:3397-3403

41. Stitt A, Gardiner TA, Alderson NL et al (2002) The AGE inhibitor pyridoxamine inhibits development of retinopathy in experimental diabetes. Diabetes 51:2826-2832

42. Degenhardt TP, Alderson NL, Arrington DD et al (2002) Pyridoxamine inhibits early renal disease and dyslipidemia in the streptozotocin-diabetic rat. Kidney Int 61:939-950

43. Machado AP, Pinto RS, Moyses ZP, Nakandakare ER, Quintao EC, Passarelli M (2006) Aminoguanidine and metformin prevent the reduced rate of HDL-mediated cell cholesterol efflux induced by formation of advanced glycation end products. Int $\mathrm{J}$ Biochem Cell Biol 38:392-403

44. Hoang A, Murphy AJ, Coughlan MT et al (2007) Advanced glycation of apolipoprotein A-I impairs its anti-atherogenic properties. Diabetologia 50:1770-1779

45. Brown BE, Mahroof FM, Cook NL, van Reyk DM, Davies MJ (2006) Hydrazine compounds inhibit glycation of low-density lipoproteins and prevent the in vitro formation of model foam cells from glycolaldehyde-modified low-density lipoproteins. Diabetologia 49:775-783

46. Forbes JM, Yee LT, Thallas V et al (2004) Advanced glycation end product interventions reduce diabetes-accelerated atherosclerosis. Diabetes 53:1813-1823

47. Zieman SJ, Melenovsky V, Clattenburg L et al (2007) Advanced glycation endproduct crosslink breaker (alagebrium) improves endothelial function in patients with isolated systolic hypertension. J Hypertens 25:577-583

48. Bolton WK, Cattran DC, Williams ME et al (2004) Randomized trial of an inhibitor of formation of advanced glycation end products in diabetic nephropathy. Am J Nephrol 24:32-40 
49. Freedman BI, Wuerth JP, Cartwright K et al (1999) Design and baseline characteristics for the aminoguanidine Clinical Trial in Overt Type 2 Diabetic Nephropathy (ACTION II). Control Clin Trials 20:493-510

50. Vasan S, Zhang X, Kapurniotu A et al (1996) An agent cleaving glucose-derived protein crosslinks in vitro and in vivo. Nature 382:275-278

51. Yang S, Litchfield JE, Baynes JW (2003) AGE-breakers cleave model compounds, but do not break Maillard crosslinks in skin and tail collagen from diabetic rats. Arch Biochem Biophys 412:42-46
52. Thornalley PJ, Minhas HS (1999) Rapid hydrolysis and slow alpha,beta-dicarbonyl cleavage of an agent proposed to cleave glucose-derived protein cross-links. Biochem Pharmacol 57:303307

53. Nanjee MN, Crouse JR, King JM et al (1996) Effects of intravenous infusion of lipid-free apo A-I in humans. Arterioscler Thromb Vasc Biol 16:1203-1214

54. Chetiveaux M, Lalanne F, Lambert G, Zair Y, Ouguerram K, Krempf M (2006) Kinetics of prebetal HDL and alphaHDL in type II diabetic patients. Eur J Clin Invest 36:29-34 